\title{
Handwriting Quality Analysis using Online-Offline Models
}

This paper was downloaded from TechRxiv (https://www.techrxiv.org).

\section{LICENSE}

CC BY 4.0

SUBMISSION DATE / POSTED DATE

06-10-2020 / 12-10-2021

CITATION

Hamdi, Yahia; Akouaydi, Hanen; Boubaker, Houcine; Alimi, Adel (2020): Handwriting Quality Analysis using Online-Offline Models. TechRxiv. Preprint. https://doi.org/10.36227/techrxiv.13058366.v3

$\mathrm{DOI}$

10.36227/techrxiv.13058366.v3 


\title{
Handwriting Quality Analysis using Online- Offline Models
}

\author{
Yahia Hamdi i, ${ }^{, *}$ Hanen Akouaydi ${ }^{1}$ Houcine Boubaker ${ }^{1}$ \\ Adel M. Alimi ${ }^{1,2}$
}

Received: date / Accepted: date

\begin{abstract}
This work is part of an innovative e-learning project allowing the development of an advanced digital educational tool that provides feedback during the process of learning handwriting for young school children (three to eight years old). In this paper, we describe a new method for children handwriting quality analysis. It automatically detects mistakes, gives real-time on-line feedback for children's writing, and helps teachers comprehend and evaluate children's writing skills. The proposed method adjudges five main criteria: shape, direction, stroke order, position respect to the reference lines, and kinematics of the trace. It analyzes the handwriting quality and automatically gives feedback based on the combination of three extracted models: Beta-Elliptic Model (BEM) using similarity detection (SD) and dissimilarity distance (DD) measure, Fourier Descriptor Model (FDM), and perceptive Convolutional Neural Network (CNN) with Support Vector Machine (SVM) comparison engine. The originality of our work lies partly in the system architecture which apprehends complementary dynamic, geometric, and visual representation of the examined handwritten scripts and in the efficient selected features adapted to various handwriting styles and multiple script languages such as Arabic, Latin, digits, and symbol drawing. The application offers two interactive interfaces respectively dedicated to learners, educators, experts or teachers and allows them to adapt it easily to the specificity of their disciples. The evaluation of our framework is enhanced by a database collected in Tunisia primary school with 400 children. Experimental results show the efficiency and robustness of our suggested framework that helps teachers and children by offering positive feedback throughout the handwriting learning process using tactile digital devices.
\end{abstract}

\section{Keywords}

Multi-lingual Handwriting quality analysis; Beta-Elliptic Model; Fourier Descriptor model; CNN, SVM.

\section{Declarations}

\section{Compliance with Ethical Standards}

Funding: This study was funded by the Ministry of Higher Education and Scientific Research of Tunisia (grant number LR11ES4).

\section{Conflict of Interest}

The authors declare that they have no conflict of interest.

\section{Ethics approval}

This article does not contain any studies with human participants or animals performed by any of the authors.

* Corresponding author: Yahia Hamdi E-mail: yahia.hamdi@regim.usf.tn Authors E-mail \{yahia.hamdi, hanen.akouaydi, houcine.boubaker, adel.alimi\}@regim.usf.tn

${ }^{1}$ REGIM-Lab.: REsearch Groups in Intelligent Machines, University of Sfax,

ENIS, BP 1173, Sfax, 3038, Tunisia.

${ }^{2}$ Department of Electrical and Electronic Engineering Science, Faculty of Engineering and the Built Environment, University of Johannesburg, Johannesburg, South Africa 


\section{Introduction}

Nowadays, many studies have been developed on handwriting learning topics. It is considered as one of the current challenges in multi-script handwriting analysis such as Arabic and Latin due to the variability of their characteristics and writing styles. After a comparative study, Jolly et al. [1] have shown that the number of children trained on digital devices [2] has improved significantly compared to others trained on paper especially in fluency terms. In this context, this paper deals with the problem of handwriting quality analysis. It consists of developing an educational tool allowing to help teachers and children from primary schools in the learning process and handwriting evaluation.

More precisely, this study contributes to the innovative learning project that takes advantage of the digital devices in primary schools with multiplesequence traces (Arabic and Latin letters, cursive Arabic word, symbol drawing, and digits) on two main aspects.

- It provides a simple interface that allows children to choose the target sequence and works independently with real-time and online feedback.

- It helps teachers assess children's writing skills and determine their difficulties.

The main objective of our project is to provide an advanced digital writing experience at school by using digital devices (tablets and tactile). The validation of the research project is founded on experiments conducted on primary school children's in Tunisia (see Fig.1) who were drawing the block of Arabic, Latin, Urdu and Persian letters, Arabic words, and symbols as illustrated in and Fig.2. The handwriting quality analysis depends generally on readability (shape), order, and direction aspects. Indeed, at the beginning of the learning stage, it is more important to have powerful constraints on order and direction aspects to obtain correct handwriting coordination. However, shape represents the main aspect to be evaluated during the learning process. The score given to the shape aspect decreases consecutively with symbol deformation. Contrary, scores related to direction and order aspects correspond to a binary decision. In fact, education experts do not forever agree on alone writing convention but think that children must follow well-defined rules to know how to write correctly any symbol.

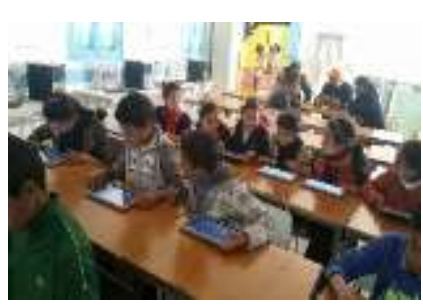

(a)

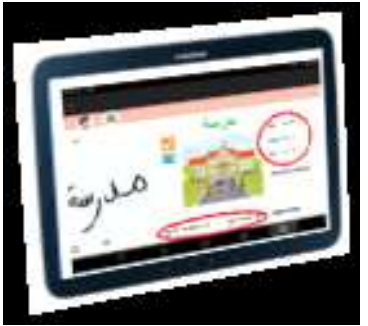

(b)

Fig. 1. Experiment in-class of the proposed project (a) with tactile tablet devices about the analysis of supported scripts (b).

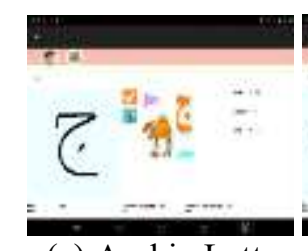

(a) Arabic Letter

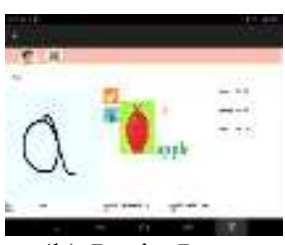

(b) Latin Letter

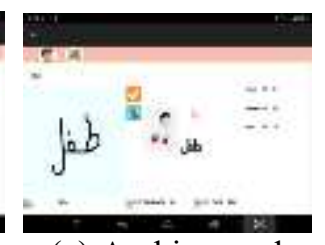

(c) Arabic word

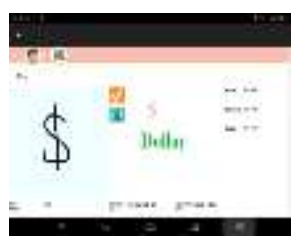

(d) Symbol

Fig. 2. The online handwriting analysis framework enables children to be autonomous during the learning and analysis tasks of letters (a, b), words (c), and symbols (d).

One of the most important problems of handwriting quality analysis is the selection of the relevant set of features that allows characterizing such criteria. For instance, fuzzy histograms of direction and orientation, histograms of points are used by [3] to identify the finely shape and direction of letters. Recently, deep learning technologies have proven highly successful in different topics of computer vision such as pattern recognition. $\mathrm{CNN}$ is one of the most widespread kinds of neural networks applied for action recognition [4], [5], 
handwriting recognition [6], [7], etc. It is considered as a powerful feature extraction model that extracts automatically multiple low and high features [8], [9]. This is the reason why, in this framework, we focus on CNN to extract efficient deep learning features allowing to characterize and analyze online children's handwriting shape with different writing style.

The main contributions of our work consist of developing a framework for multi-scripts handwriting quality analysis and giving online feedback regards to reference models. We have employed five main criteria (shape, direction, order, kinematics, position with respect to the reference lines) with specific types of features (see section 5) that allow the characterization of the children writing style: BEM, FDM, and deep learning-based CNN feature. The former is used for elementary stroke segmentation of the online handwriting trajectory. It apprehends among the dynamic features related to the motion, the geometry of the elementary stroke, and their sequence time. The second analysis feature allows us to describe the overall geometry of the trace based on the undulations of its curvature function. Finally, CNN model is utilized for the perceptual capture of the post-drawing trace.

This paper is organized as follows. Section 2 depicts the state of the art of some works in handwriting quality analysis. In section 3, we introduce the proposed framework architecture and used criteria analysis. Section 4 summarizes the preprocessing techniques that are used in our work. The handwriting feature extraction models and comparison engines are described in section 5. The evaluation of our system and the experimental results are discussed in Section 6. Finally, section 7 provides a conclusion and future work.

\section{Related work}

Handwriting quality analysis is related to readability (shape) and kinematics in the literature [10]. Indeed, handwriting readability corresponds to the shape of the letter, while, kinematics is focused on the writing process (eg, direction, order, fluidity) to be effective because writing is a fundamental skill needed to learn and use knowledge.

Descriptions of many criteria that represent the critical components of handwriting readability are discussed in [11]. It includes slant, size, the degree of line straightness, spacing, shape (letter form), and the general merit of the writing. The authors deduce that computer analysis is more sensitive, accurate, and reliable than subjective analysis, although they observe that present practical applications are still restricted.

There are generally two types of applications resulting from handwriting analysis: education and medical systems. Guinet et al. [12] interested in the kinematic aspects (i.e. duration, velocity, fluency) to distinguish handwriting pathologies. Jolly et al. [1] focused on handwriting velocity in order to indicate disorders of development coordination. Various features (e.g. pen lift duration, peak velocities, and the number of strokes per letter) are used by [13] to investigate handwriting kinematics of children on digital devices like tablets. It also shows that four kinematic domains: (automation and motor planning plan, velocity, spatial arrangement) are necessary for the best handwriting quality. Another educational system for Chinese characters is introduced by [14]. It was identified by three types of errors such as stroke production errors (i.e. broken strokes, stroke reversal, ..etc), stroke relationship errors (e.g. length and position), stroke sequence errors (i.e. wrong sequencing of components, wrong stroke sequences in a component). Falk et al. [15] adopt five primitives (i.e. size, form, space, alignment, and legibility) to quantify children's writing skills. More generally, the kinematic aspect is used mainly by medical systems like [1], [12] by opposition to educational systems [13], [14] which is particularly interested in legibility features.

Recently, Simonnet et al. [3] proposed a multi-criteria approach for Latin handwriting quality analysis. In this work, the children's writing is evaluated with regards to reference models using intra-class and inter-class scores. Indeed, a multi-criteria score describes the legibility (shape) and kinematic (order and direction) aspects for children, according to the teacher expectations. In Simonnet et al. [16] the authors introduced an explicit segmentation approach for handwritten cursive word evaluation. They start by identifying the 
primary segmentation hypotheses to reduce error propagation by adding a verification step through supervision. Next, they extract the letter hypotheses based scoring followed by word hypothesis extraction and evaluation by combining elastic matching and writing analysis scores. More recently, the authors [17] used the fusion of multiple CNN channels networks for online children handwriting recognition. They converted the online signal into multiple image channels taking into account the dynamic information to improve the performance of a CNN. Krichen et al. [18], proposed an orthographic analysis approach of children handwritten script in a dictation context based on two strategies. The first strategy uses the explicit ground-truth of the input word as prior knowledge of the handwriting analysis process and to recover the written word. The second consists of generating hypotheses phonetically similar to the dictated instruction, which will serve as probable approximations of the written word, to cover any possible orthographic mistakes by the pupil.

\section{Handwriting quality evaluation method}

The present framework proposes an algorithm for analyzing the quality of children's handwriting. It consists of the adoption of multiple evaluation criteria and the application of powerful models to extract effective and complementary features vectors. Our goal is to help teachers evaluate handwriting and provide remedial feedback to children's schools during the learning process. As shown in Fig.3, the handwritten sequences are online signals captured with digital devices that have undergone a pre-processing step. Then, five main criteria are employed to evaluate the quality of handwriting based on the combination of three models of handwriting modeling: BEM, FDM, and CNN. Finally, a significant appreciation score is established for each criterion related to reference models (database of samples) based on SD-DD and SVM engines respectively.

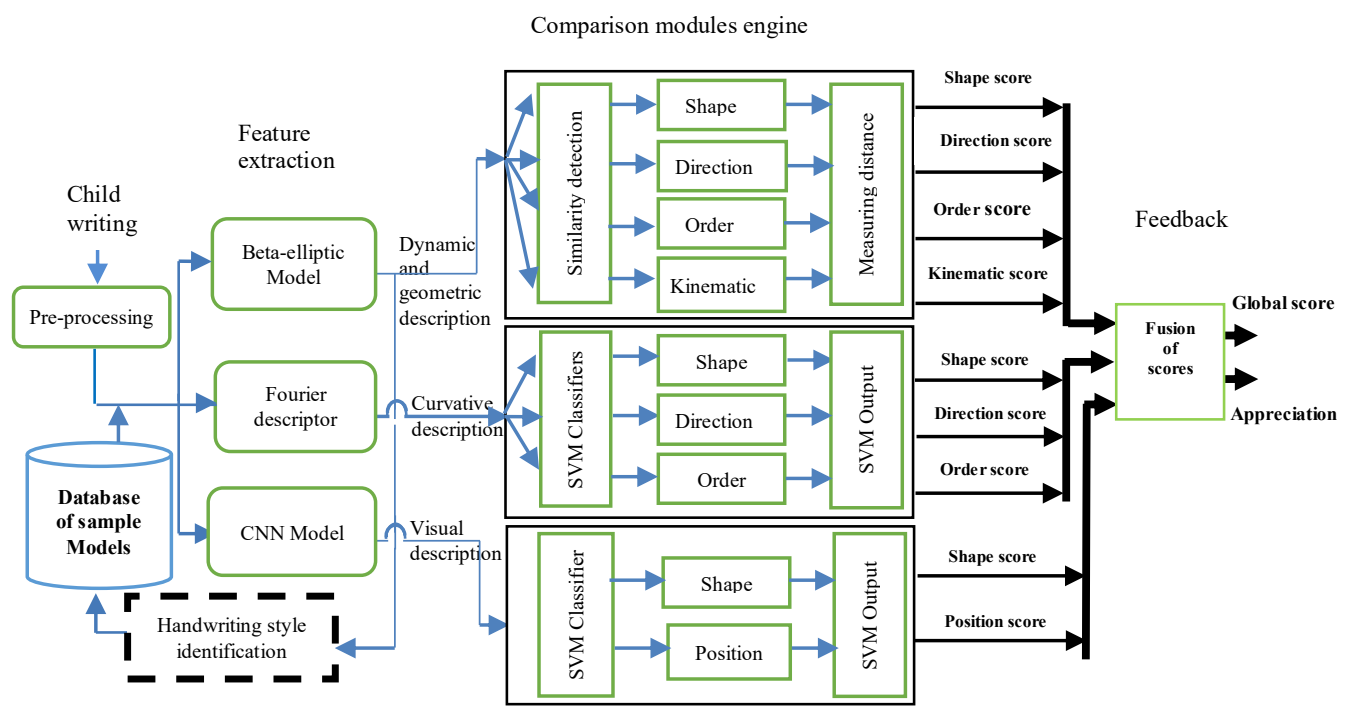

Fig. 3. Flowchart of the proposed framework.

\subsection{The evaluation criteria}

In this section, we briefly outline the list of evaluation criteria adopted by the proposed system for handwriting quality analysis.

\subsubsection{Graphical shape}

The handwriting shape criterion is the main component of the handwriting quality assessment process. It corresponds to the fusion between the levels of graphical fidelity and readability of the produced writing taking into account the size and alignment on the guideline. Some geometric parameters such as the locations of a set of trajectory guide points that includes the segments endpoints and their intermediate points corresponding to velocity or curvature local extremums (see Fig.4.a and Fig.4.b for the correct and incorrect shape of the Arabic character ' $b$ ' respectively). The global geometry of the trajectory 
segments and their curvature functions are also considered among the properties that characterize the shape criterion.

\subsubsection{Strokes order and direction}

We suggest that the correct writing order of the sequence (letter, word, symbol) corresponds to the correct succession of the composed elementary strokes. Indeed, each script has its own properties distinguished from others. For instance, the Arabic language has several characters that contain dots (i.e: ب, ) or small marks (s) and other without dots $(\varepsilon, \tau)$. We always start with the main corps before the diacritics. Fig.4. d shows an example of wrong order detected by our system in which the segment '" is written before the occlusion 'صـ'. Also, the correct direction is identified using specifics features such as start and endpoint positions, and their trajectory tangents directions. (see Fig.4.c).

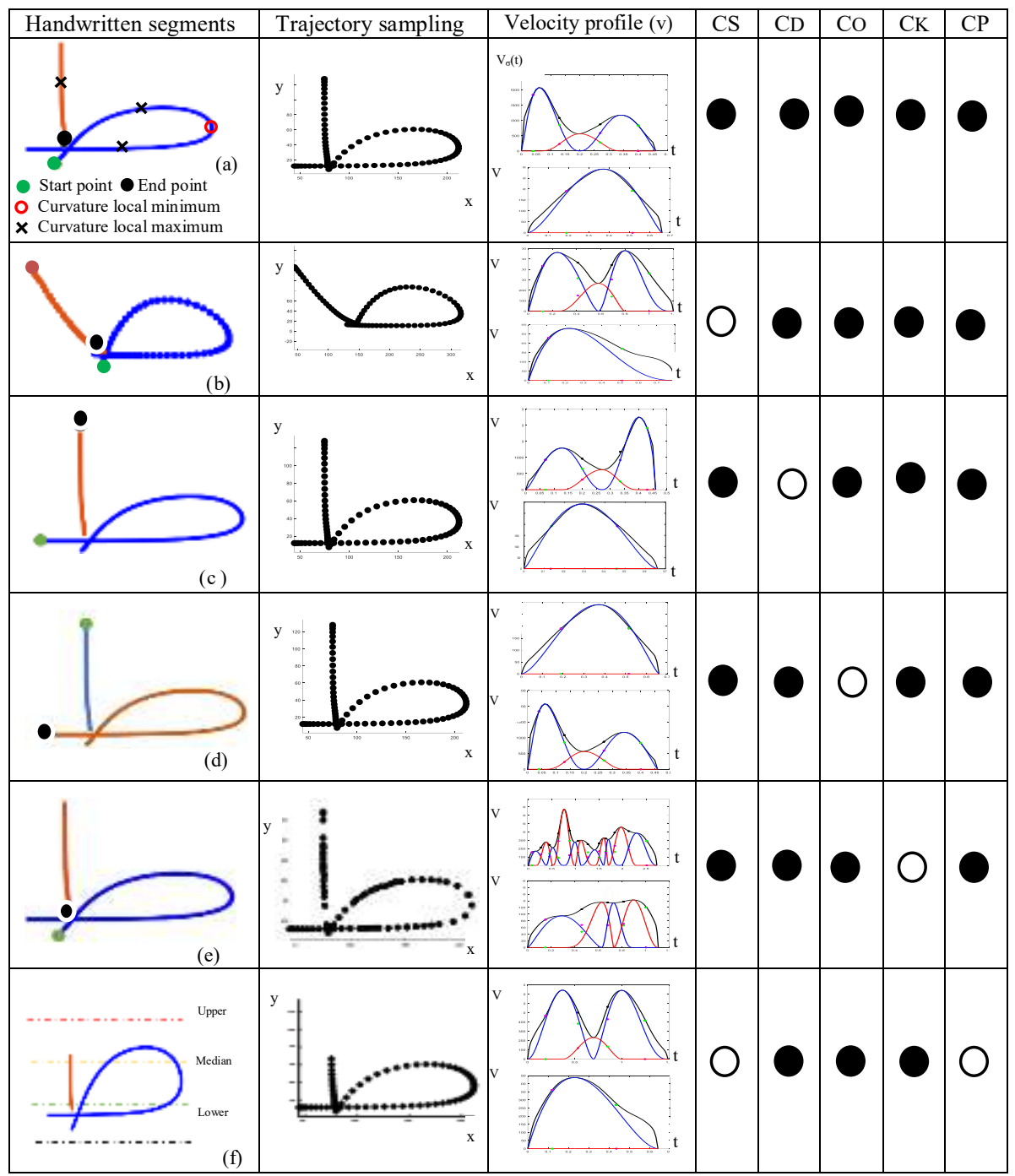

$C S$ : correct shape, $C D$ : correct direction, $C O$ : correct order, $C K$ : correct kinematic, $C P$ : correct position to respect reference line.

Fig.4. Presentation of the evaluation criteria: The first column represents the handwritten segments, while the second describes the temporal trajectory sampling. The third column shows the velocity profile of the correspondent trajectory sample. The last five columns depict respectively the correctness degree (black and white points for commonly correct and wrong criterion respectively) of the reported criteria.

\subsection{3 kinematics of the trace}

The handwriting movement is considered as one of the most aspects of handwriting modeling systems that synthesis the neurophysiologic characteristics of the involved organs (muscles, hand, arm, finger joint, ...). A lot of research examines the kinematic of the handwriting trajectories and 
concentrates most of the information to be extracted in velocity profiles. According to this description, we believe that the concept of kinematics is very important to evaluate the dynamism of children 's writing. As shown in Fig.4.e, we notice a variation between regular and irregular writing kinematics, since this causes some diversity in the interpolation points of the trajectory.

\subsubsection{Respect of the reference lines (trace position)}

At the beginning of the school children's learning process, the baseline aspect is needed to learn and assess the competence of linear cursive writing. It is one of the most significant elements of handwriting analysis. It helps to determine how to deal with the combination of cognitive, social, and instinctual drives. Based on this criterion, the online signal can be decomposed into three main zones: Median, Upper, and Lower (see Fig.4.f) which allows the child to distinguish between the different forms of letters: those written on, below, and above the baseline.

\subsection{Selection of the pertinent parameters}

The evaluation of the quality of learners' writing tests depends on the five criteria presented above. The proportional progression of scores for all criteria during the learning process is not guaranteed since the learner often tends to focus on one or two criteria at the same time. On the other hand, the considered diversification of the evaluation criteria requires analogously a diversity of parametric models. Thus, the aim is to quantify the evaluation outcome of each separate criterion by computing its different associated parameters in order to deliver a final information report to the learner or the expert.

To ensure a strong consistency between the different analysis criteria, we applied the combination of three complementary models. In fact, we take advantage of BEM characteristics to apprehend the dynamic and graphical features of the elementary beta strokes (delimited by two successive extremums of velocity or curvature radius) composing the hand drawing trajectory. Further, the FDM and CNN models are used to describe respectively the variation of the trajectory curvature function and the final view obtained post-drawing.

However, to assess the compliance with the two criteria direction and order, we set aside the offline CNN model to keep only the BEM parameters as well as the FDM curvature model. Likewise, to evaluate the accordance with the kinematics criterion, we relied only on the dynamic features subset of BEM to compare the velocity profile of the test script to existing models recorded by an expert (voluntary teachers). The details of the different models employed are described in section 5 .

\section{Pre-processing}

The handwriting trajectories are collected online via a digitizing device. It is characterized by a high variation that requires applying geometric and denoise processing steps to minimize handwriting variabilities and reduce noise. Given the raw trajectory, the low-pass filtering Chebyshev type II with a cut-off frequency of fcut $=10 \mathrm{~Hz}$ is used to mitigate the effect of noise and errors due to temporal and spatial quantification introduced by the acquisition system as shown in Fig.5. The value of cut-off frequency results from a compromise between the conservation of the handwriting undulations produced by young writers and the elimination of those due to the pathologic tremor of other older writers [19].

The horizontal fulcrums level of character handwriting decomposes their drawing area into three zones namely upper, core, and lower regions respectively. Consequently, a procedure for normalizing the size of the handwriting is applied to adjust its height to a fixed value $\mathrm{h}=128$, while keeping the same ratio length/height [20], [21]. Both the preprocessing technique are used and tested for the supported scripts. 


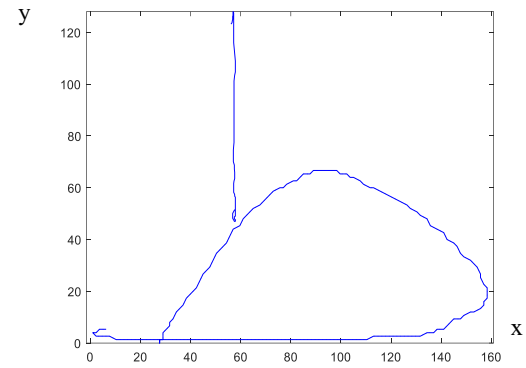

(a)

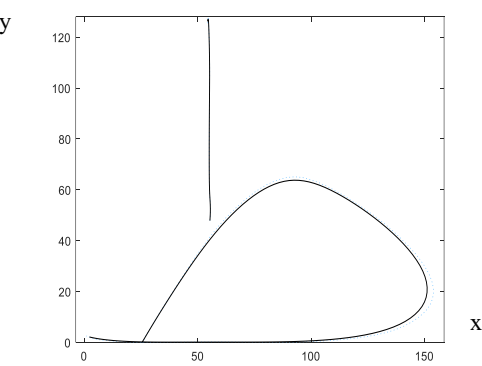

(b)

Fig.5. (a) Acquired raw trajectory of the Arabic character ' $b$ ' and (b) after Lowpass filtering and smoothing.

\section{Features extraction and comparison engines}

Our developed handwriting analysis system uses a new method based on the combination of handcrafted and deep learning feature extraction models coupled with different criteria assessment engines. These modules are described in turn in this section.

\subsection{Features extraction}

Three specifics types of features are introduced in our work: BEM, FDM, and $\mathrm{CNN}$ models. We explain in this sub-section the principle of each model.

\subsubsection{Beta-elliptic model (BEM)}

The BEM derives from the kinematic Beta model with a juxtaposed analysis on the spatial profile. It considers a simple movement as the response to the neuromuscular system which is described by the sum of impulse signals [22], [23], or Beta function [24]. It consists of decomposing the online trajectory into elementary stroke based on the combination of the velocity profile (dynamic features) and static profile (geometric features) of online handwriting modeling. Further, BEM has proven highly successful in various areas of research on online handwriting like the study of handwriting recognition [25], [26], the effect of age on hand movement kinematics [27], temporal order recovery [28], [29], and writer identification [30]. To our knowledge, the BEM has not yet been used in handwriting evaluation. In fact, our idea is to exploit the efficiency of the real-time description of handwriting movements provided by this model in our context.

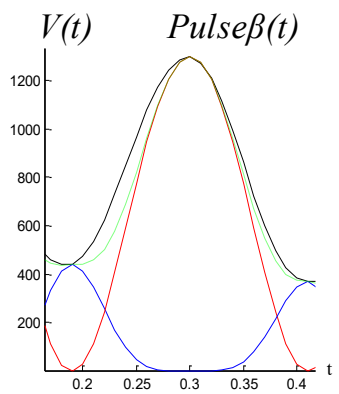

(a) Beta function

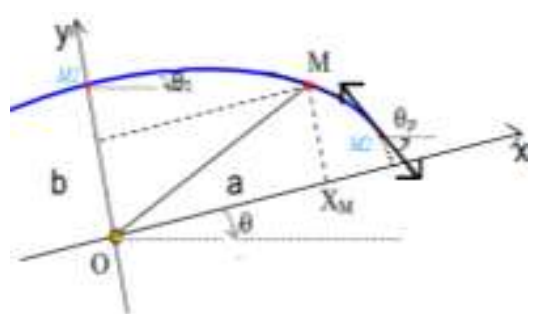

(b) Elliptic arc

Fig. 6 Online handwriting modeling using BEM.

\section{Velocity profile:}

In the dynamic profile, the curvilinear velocity curve $V \sigma\left(t_{c}\right)$ shows a signal that alternates between extremums (minima, maxima, and inflexion points) which delimit and define the number of trajectory strokes. It can be reconstructed by overlapping Beta signals where each stroke corresponds to the generation of one beta impulse (see Fig.6.a) represented by the following expression:

$$
\operatorname{pulseB}\left(t, q, p, t_{0}, t_{1}\right)=\left\{\begin{array}{l}
k \cdot\left(\frac{t-t_{0}}{\left(t_{c}-t_{0}\right)}\right)^{p} \cdot\left(\frac{t_{1}-t}{\left(t_{1}-t_{c}\right)}\right)^{q} \text { if } t \in\left[t_{0}, t_{1}\right] \\
0 \text { elsewhere }
\end{array}\right.
$$




$$
t_{c}=\frac{p^{*} t_{1}+q^{*} t_{0}}{p+q}
$$

Where, $t_{0}$ and $t_{1}$ are respectively the starting and the ending times of the generated impulse which delimiting the correspondent trajectory stroke, $t_{c}$ is the instant when the beta function reaches its maximum value as depicted in Eq. 2, $K$ called impulse amplitude, $p$ and $q$ are intermediate shape parameters.

$$
V_{\sigma}(t)=\sum_{i=1}^{n} V_{i}\left(t-t_{0 i}\right) \approx \sum_{i=1}^{n} \operatorname{pulse} \beta i\left(K_{i}, t, q_{i}, p_{i}, t_{0 i}, t_{1 i}\right)
$$

As shown in Fig. 7.a) and c), the velocity profile of the handwritten trajectory of the Arabic character 'S 'S' and Latin word 'fleur' can be reconstructed by the overlapped beta signals as described in Eq. 3. Indeed, the dynamic features give the overall temporal properties of the neuromuscular networks implicated in the motion generation of the writing process.

\section{Geometric profile:}

In the space domain, each elementary beta stroke located between two successive extrema speed times can be modeled by an elliptic arc characterized by four geometric parameters: $a, b, \theta, \theta_{p}$ (see Fig.6.b). Where $a$ and $b$ represent respectively the half dimensions of the large and the small axes of the elliptic arc, $\theta$ : the angle of the ellipse major axe inclination, and $\theta_{p}$ is the trajectory tangent inclination at the minimum velocity endpoint. These parameters reflect the geometric properties of the end effector (pen or finger) trace, dragged by the set of muscles and joints involved in handwriting.

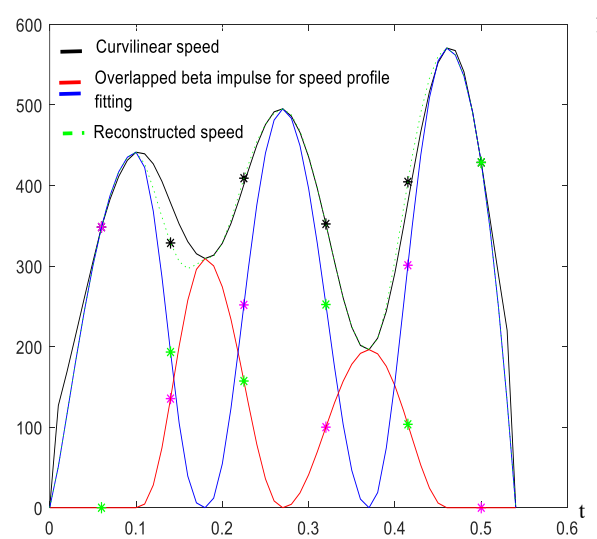

(a)

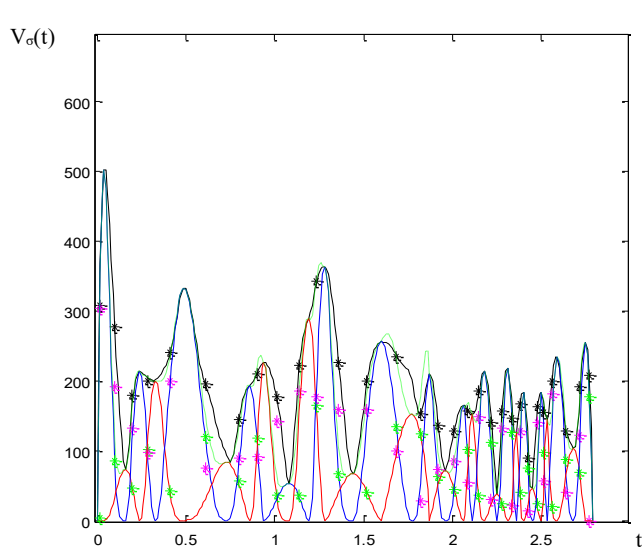

(c)

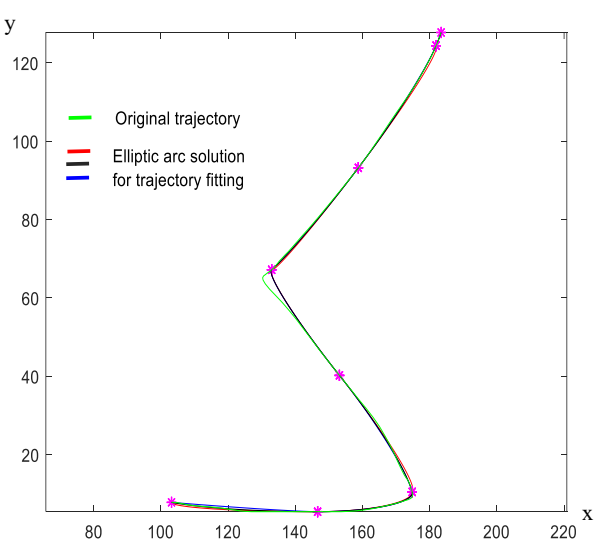

(b)

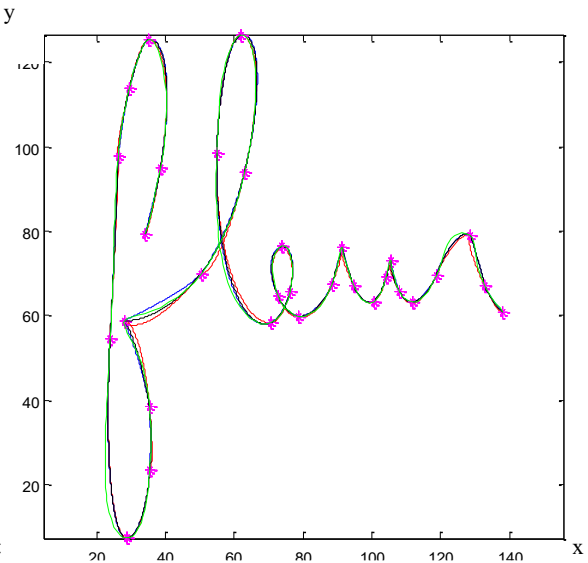

(d)

Fig.7. Online handwriting modeling of Arabic character 's' and Latin word 'fleur' with BEM. (a) and (c) describe the velocity profile, (b) and (d) represent the geometric profile.

\subsubsection{Fourier descriptor model (FDM)}

FDM is one of the most accurate tools for parametric modeling of the closed path which can be represented by a $2 \pi$ periodic signature function [31]. To 
benefit from their powerful capacity of periodic function approximation in trajectories stroke modeling, we must transform the signatures corresponding to the stroke open trajectories into periodic functions. Each segmented trajectory is represented by an angular signature modeled by a periodic sinusoidal function. As shown in Fig. 8, the chosen function as a stroke trajectory signature, describe the variation of the inclination angle $\theta_{i}$ of the trajectory tangent at a point $M_{i}$ depending on its corresponding curvilinear abscissa:

$$
{ }_{\ell-1}^{i} L_{j} \quad \text { for } \quad i=1, \ldots, 2 n
$$

Where, $d L_{i}$ represent the distance between the current point $M_{i}$ and its previous. Further, the Fourier descriptors coefficients $a_{0}, a_{j}$ and $b_{j}$ of the Fourier series that approximates the signature function $\theta_{i}=f\left(\ell\right.$ at the $8^{\text {th }}$ harmonic are computed by Eqs. (5) and (6):
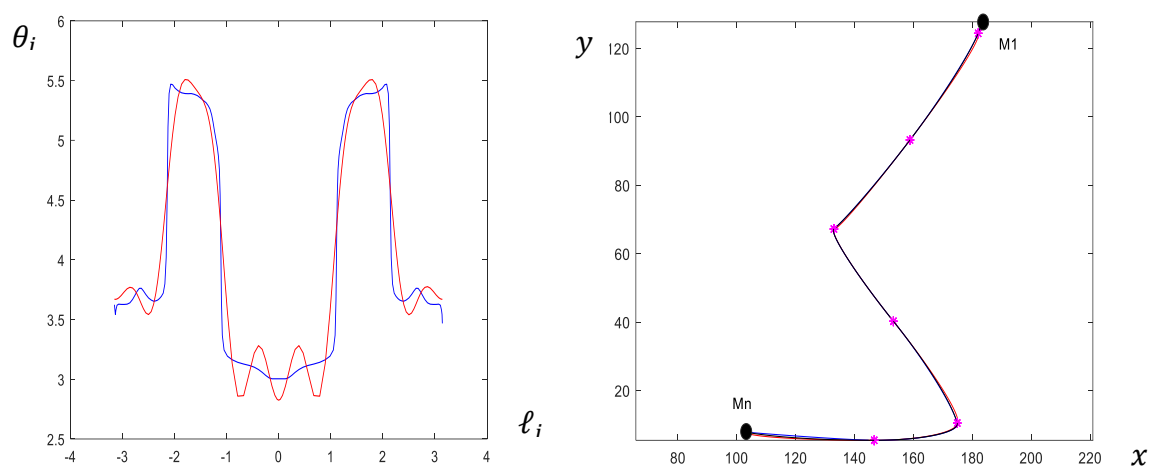

Fig.8. Signature functions approximated at the $8^{\text {th }}$ harmonic and correspondent trajectory of Arabic character "ك".

$$
\begin{gathered}
a_{0}=\frac{1}{2 \pi} \cdot \sum_{i=1}^{2 n} \theta_{i} \cdot d L_{i} \\
a_{j}=\frac{1}{\pi} \cdot \sum_{i=1}^{2 n} \theta_{i} \cdot \cos \left(j \cdot \frac{2 \cdot \pi \cdot \ell}{\ell}\right. \\
b_{j}=\frac{1}{\pi} \cdot \sum_{i=1}^{2 n} \theta_{i} \cdot \sin \left(j \cdot \frac{2 \cdot \pi \cdot \ell}{\ell}\right.
\end{gathered}
$$

To reconstruct the trajectory segment and the correspondent signature, we use the approximation function described by the following equation:

$\theta_{i}=f\left(\ell \quad \cdot \sum_{j=1}^{k}\left[a_{j} \cdot \cos \left(j \cdot \frac{2 \cdot \pi \cdot \ell}{\ell} \quad \ell \ell^{\ell}\right.\right.\right.$

\subsubsection{CNN shape model}

In this sub-section, we briefly explain the use of CNN model in our context. Inspired by the recent successes of deep learning technologies in different topics, we have used CNN architecture in the off-line bitmaps reconstructed from the online trajectory. The latter represents just the final layout of the hand drawing that skirts the chronologic style of its generation. Indeed, the choice of CNN architecture affects the performance of such a system. The first experiment consists of selecting the best CNN feature extraction. As shown in Table 1, five variations of $\mathrm{CNN}$ models were tested by changing the number of layers such as AlexNet [32], VGG-Net [33], Inception-V4 [34], CNN1, and CNN2.

After trying several settings, we chose CNN2 with 15 layers similar to Zhang et al. [35], because it is the most efficient architecture which generates a high accuracy for sequence recognition. The structure of CNN employed contains multiple convolution and max-pooling layers. We also used a recent dropout technique for selecting the best CNN feature extraction that characterizes the shape model. The input layer is of size $32 \times 32$ of gray-level image. The applied filter of convolutional layers is $3 \times 3$ with a fixed convolution stride to one. The dimension of feature maps in each convolution layer is increased gradually from 50 in layer-1 to 400 in layer-12. After three convolutional layers, a max-pooling $2 \times 2$ window with stride 2 is implemented to halve the size of feature map. 
Finally, two fully-connected layers with 900 and 200 hidden units respectively followed by the soft-max layer to obtain the feature vector that is used for classification. We trained the network using its parameters: a stochastic gradient descent 'sgdm' with momentum and mini-batch of size 100, the learning rate is 0.001 . However, we used the dropout technique only in the last layer.

Table 1. Structures of CNN feature extraction: "Conv: Conv.-Norm -ReLU layer", "Conv: filter size", and "Max pool: filter size" are the parameters of the convolution and max-pooling layers respectively. hl: hidden layer, drop: denotes the dropout technique.

\begin{tabular}{|c|c|c|c|c|c|}
\hline \multirow{2}{*}{ Structure } & AlexNet & VGG-Net & Inception-V4 & CNN1 & CNN2 \\
\hline & & & & $\begin{array}{l}\text { Conv } 3 \times 3(50) \\
\text { Conv } 3 \times 3(100) \\
\text { Max-Pool: } 2 \times 2 \\
\text { Conv } 3 \times 3(150) \\
\text { Conv } 3 \times 3(200) \\
\text { Max-Pool: } 1 \times 2 \\
\text { Conv } 3 \times 3(250) \\
\text { Conv } 3 \times 3(300) \\
\text { Max-Pool: } 2 \times 2 \\
\text { Conv } 3 \times 3(350) \\
\text { Conv } 3 \times 3(400) \\
\text { Max-Pool: } 2 \times 1 \\
\text { Conv } 3 \times 3(512)\end{array}$ & $\begin{array}{l}\text { Conv } 3 \times 3(50) \\
\text { Conv } 3 \times 3(100) \\
\text { Conv } 3 \times 3(100) \\
\text { Max-Pool: } 2 \times 2 \\
\text { Conv } 3 \times 3(200) \\
\text { Conv } 3 \times 3(200) \\
\text { Conv } 3 \times 3(200) \\
\text { Max-Pool: } 2 \times 2 \\
\text { Conv } 3 \times 3(300) \\
\text { Conv } 3 \times 3(300) \\
\text { Conv } 3 \times 3(300) \\
\text { Max-Pool: } 1 \times 2 \\
\text { Conv } 3 \times 3(400) \\
\text { Conv } 3 \times 3(400) \\
\text { Conv } 3 \times 3(400) \\
\text { Max-Pool: } 2 \times 1 \\
\text { \#hl:900, drop:0.2 } \\
\text { \#hl:200, drop:0.2 } \\
\text { \#units: } 512\end{array}$ \\
\hline Accuracy & $97.18 \%$ & 97.05 & $97.90 \%$ & $93.27 \%$ & $98.14 \%$ \\
\hline
\end{tabular}

\subsection{Comparison engines}

In this section, we introduce the comparison engine methods used to analyze the children's handwriting quality. Indeed, the objective is to compare the 'test' samples with three correct samples known as 'models', existing in the training database, based on the complementary extracted feature vectors.

\subsubsection{BEM based on SD-DD Method}

As mentioned previously, the BEM describes a set of dynamic and geometric features designed for online handwriting trajectory modeling by breaking it into $N$ elementary strokes delimited by velocity extremums. Based on this idea, the comparison of the test sample that is represented by a set of beta stroke $T=\left\{T_{l}\right.$, $\left.T_{2}, \ldots, T_{N}\right\}$, with the acquired model samples $M=\left\{M_{1}, M_{2}, \ldots, M_{m}\right\}$ is made between their respective strokes using a similarity detection (SD) and dissimilarity distance (DD) measure. This method uses the algorithm 1 that makes it possible to detect for each trajectory beta stroke $T_{i}$ of the 'test' sample, the most similar stroke $M_{j}$ ' of the model sample indexed in a neighborhood around its current index $i$. Then, it calculates the DDs distance between these two strokes $T_{i}$ and $M_{j}$.

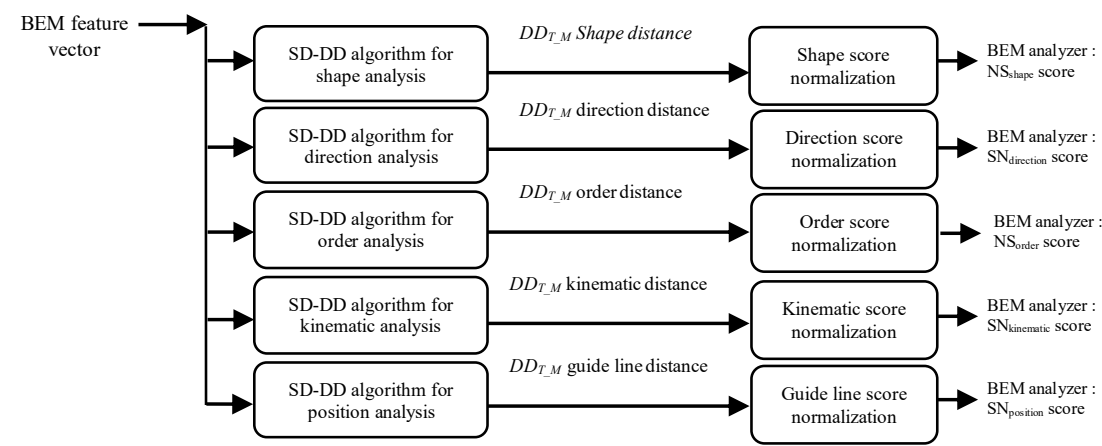

Fig.9. BEM comparison engine. 
The DD between a given test sample and the model samples noted $D D_{T_{-} M}$ is obtained by the average of the DDs of all beta strokes composing the 'test' sample in comparison with those of the model samples and vice versa.

For each evaluation criterion, the selection of the most relevant BEM parameters is performed by using a weighting selector vector $S_{p}$ of the same size as the BEM vector $V_{B E M}$ by multiplying the latter by $S_{p}$ as described in Eq.8.

$$
T_{i}=V_{B E M i} * \mathrm{~S}_{p}
$$

For the Shape criterion, all the geometric parameters of BEM are considered at weight 1 while the dynamic component is attenuated by a weighting coefficient equal to 0.2 and vice versa for the kinematic criterion. For both order and direction criteria, the most weighted BEM parameters are the coordinates of the trajectory beta stroke endpoints. Fig. 9 shows the handwriting analysis process of each criteria using BEM model and SD-DD algorithm.

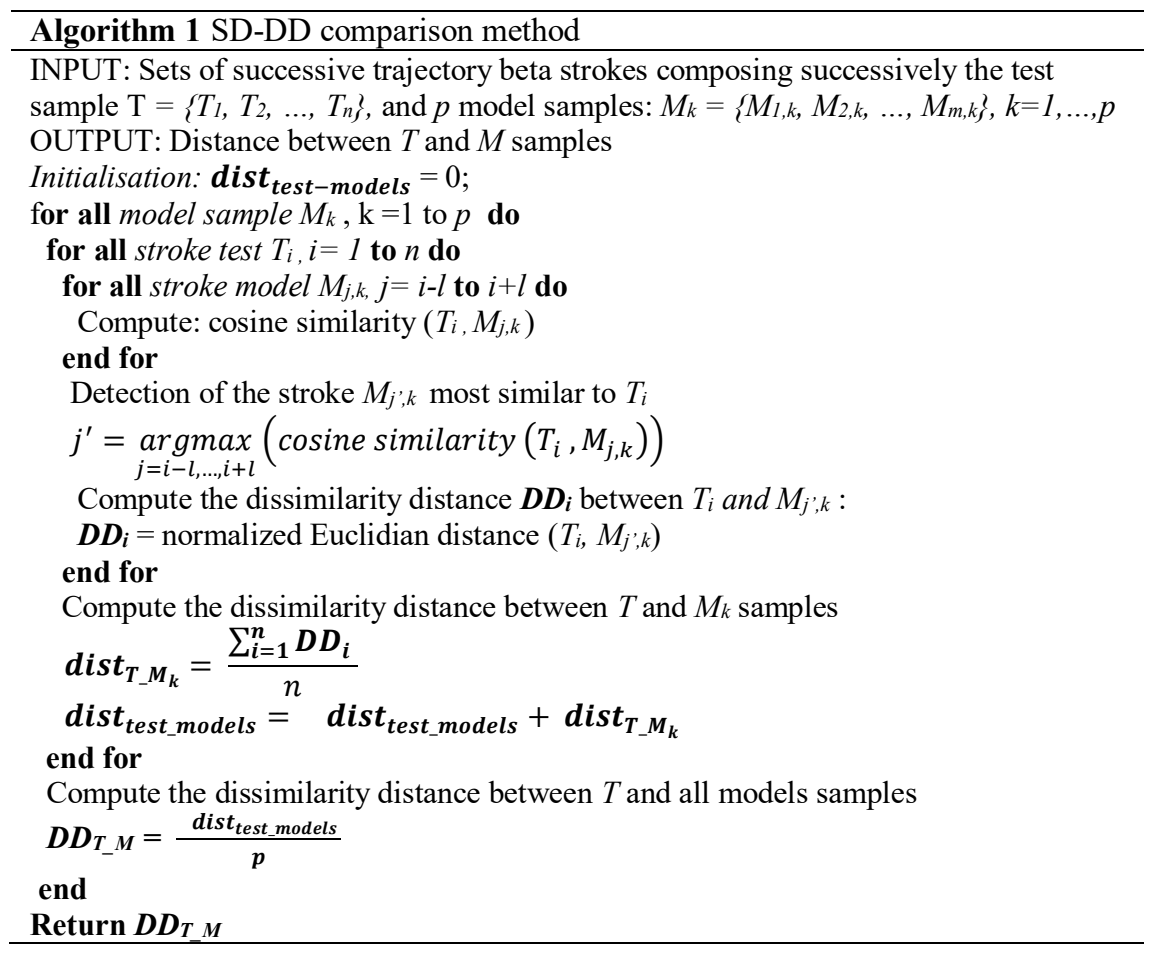

\subsubsection{SVM engine}

SVM is considered a powerful tool for linear and nonlinear classification based on a supervised learning algorithm. It has shown high success in many practical applications such as pattern recognition [36]. Contrary to traditionally artificial neural networks, the basic formulation of SVM is the structural risk minimization instead of empirical risk. As shown in Fig 10.a, SVM is mostly used to determine an optimal separating hyper-plane by adopting a novel technique that maps the sample points into a high-dimensional feature space using a nonlinear transformation. It was originally designed to solve binary classification problems. However, it can be employed also to solve multiclass problems (see Fig.10.b) using several methods such as one-versus-all [37].
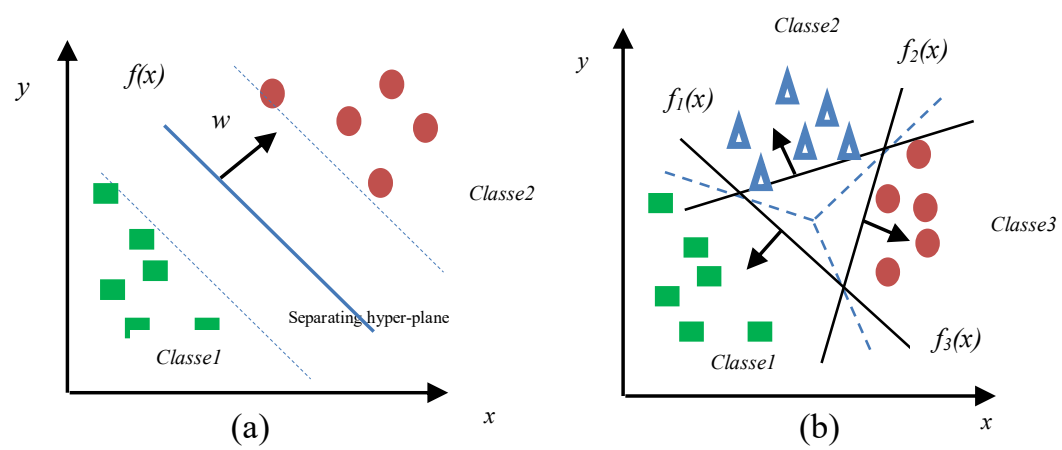

Fig.10. Principle of SVM. (a) two-class hyper-plane example, (b) one-versus-all method.

In our work, we have used multiple 'two-class' SVMs classifiers for labeling test 
sample $T_{i}$ in appropriate class like [correct shape / wrong shape], [correct order / wrong order], [correct direction / inversed direction] respectively.

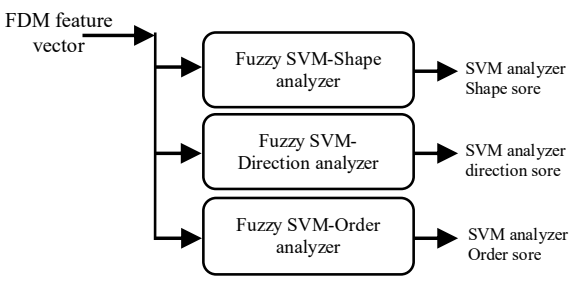

(a)

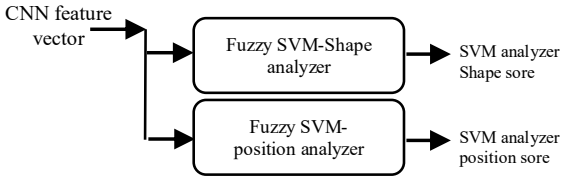

(b)

Fig.11. Handwriting analysis using SVM engine. (a) comparison using FDM model, (b) comparison using CNN feature.

After classifiers training, a crisp labeling of the test sample can be obtained by examining a sign of the output $h\left(T_{i}\right)=\left(\left\langle w, T_{i}\right\rangle+\mathrm{b}\right)$ where $h(x)=0$, defines the hyperplane separating the two classes. To obtain fuzzy membership output affecting the test sample to a couple of $C_{1}, C_{2}$ of given classes, we transform the output of each classifier $h\left(T_{i}\right)=\left(\left\langle w, T_{i}\right\rangle+b\right)$ into estimated probability $P\left(C_{j} \mid T_{i}\right)$ using the parametric approach proposed by Platt [38]. The probability $P\left(C_{j} \mid T_{i}\right)$ is modeled by a sigmoid function as described in Eq. 9:

$$
\text { ¿い上 }-\frac{1}{1+e^{A h(T)+B}}
$$

where A and B are parameters calibrated the output probability between 0 and 1 . Fig. 11.a) and Fig. 11.b) show the mechanism of handwriting analysis using a feature vectors extracted from FDM and CNN models.

\section{Experimental results and discussion}

This section presents experimental results that are performed on handwriting quality analysis. Datasets are firstly presented followed by the conducted experimental setup. Finally, the carried out experiments and obtained results, as well as a discussion, are explained.

\subsection{Dataset}

The dataset used to validate the efficiency and robustness of our system is collected by 400 children aged four to eight in different Tunisian primary schools. The constructed dataset includes three subsets. Set 1 is dedicated to

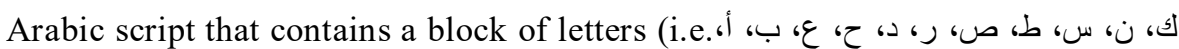

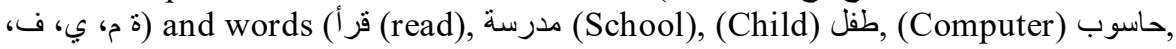

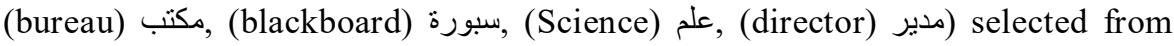
the primary textbook. Set 2 comprises some samples of Latin characters (i.e. A, C, D, E, H, I, L, M, N, O, P, R, U). Also, we have evaluated our system with other observations from set 3 that contains Urdu characters (i.e. $\stackrel{\jmath}{\jmath}, j, \xi, \Sigma,<$ ), Persian characters (i.e. $₹, \hookrightarrow, \xi, \zeta)$, and some symbols such as $(\&, \$, £, \Omega$ ). Indeed, 50 correct samples for each sequence (letters, words, symbols) are utilized for training dataset with a few samples of incorrect order and direction. However, to fully validate order and direction classifiers aims to increase the dataset, we generated additional negative samples (wrong order/direction) from positive samples by synthetically changing the order and direction. Similarly, we applied distortion technique [39] by modifying some parameters such as the angle of inclination of the trajectory, the baselines and smoothing in order to construct other positive/negative shapes by generating more training samples. The employed dataset is divided into a training set of 100.000 samples and a test set of 30.000 samples.

\subsection{Setup}

To study the impact of the already mentioned models and their combination in handwriting quality analysis, we have designed four groups of experiments. The first one is based on BEM using SD-DD comparison method. The second and third tests are realized respectively on FDM and CNN based on SVM classifier. Finally, the fourth test is founded on the fusion of the three tests.

BEM with SD-DD: The BEM is used to extract a set of pertinent features to 
characterize the five analysis criteria. The system determines for each criterion two thresholds: $T_{C C}$ and $T_{C W}$ delimiting respectively three evaluation zones: certainly correct $(C C)$, Fuzzy $(F)$, and certainly wrong $(C W)$. As described in Eqs. (10) and (11), these thresholds are computed after statistical analysis of two distributions distances $\left\{D D_{C_{-} M}\right\}$ and $\left\{D D_{W_{-}}\right\}$separating the model samples from correct and wrong samples respectively.

$$
\begin{gathered}
T_{C C}=\min \left(Q_{\left\{D D_{C_{-} M}\right\}}\left(u_{\max }\right), Q_{\left\{D D_{W_{-} M}\right\}}\left(u_{\min }\right)\right) \\
T_{C W}=\max \left(Q_{\left\{D D_{C_{-} M}\right\}}\left(u_{\max }\right), Q_{\left\{D D_{W_{-} M}\right\}}\left(u_{\min }\right)\right)
\end{gathered}
$$

Where $Q_{\{D D\}}(u)$ is the value of the quantile function of the $\{D D\}$ distribution at a cumulative probability of $u$ percent. $u_{\max }$ and $u_{\min }$ are adjustable cumulative probabilities that limit the substantial part of the distribution $D D$ fixed empirically to $96 \%$ and $4 \%$ respectively.

A first normalized score $N S_{I}$ is assigned to the test sample relying on the correct partition using Eq. 11.

$$
\left\{\begin{array}{l}
\text { if } D D_{T_{-} M}<T_{C C} \Rightarrow N S_{1}=1 \\
\text { elseif } D D_{T_{-} M}>T_{C W} \Rightarrow N S_{1}=0 \\
\text { else } N S_{1}=\frac{T_{C W}-D D_{T_{-}}}{T_{C W}-T_{C C}}
\end{array}\right.
$$

Similarly, we calculated the $D D_{T_{-} W}$ distance separating the test sample from the set of wrong samples which represents the most common errors before converting it to a normalized score noted $N S_{2}$ between 0 and 1 analogously to Eq.12. As described in Eq. 13, the final score $N S$ is computed as the average between $N S_{1}$ and the complement of $N S_{2}$.

$$
N S=\frac{N S_{1}+\left(1-N S_{2}\right)}{2}
$$

FDM and CNN based on SVM classifier: In this part, we have initially extracted the feature vectors using FDM and CNN models. Thereafter, these later are used as input for the multiclass SVM with RBF kernel function for making classification step. The SVM model allows characterizing such criteria: shape, direction, and order using FDM and only shape and position criteria with CNN model relying on the classifier confidence score.

Combined models: The fourth experiment evaluated the impact of the hybrid results of the already mentioned models. The final score assigned for each criterion is calculated as a weighted average of the scores assigned by each separate subsystem weighted by its correct classification rate (CCR).

\subsection{Results}

Our system considers three evaluation levels: a global description level which allows to classify the test script into six classes identified by a label $L_{i c}$ and a digital index $i_{c}:\left(i_{c}=1, L_{1}=\right.$ correct; $i_{c}=2, L_{2}=$ wrong shape; $i_{c}=3, L_{3}=$ wrong order; $i_{c}=4, L_{4}=$ wrong direction; $i_{c}=5, L_{5}=$ reference line surpass; $i_{c}=6, L_{6}=$ irregular kinematics). The second level called quantitative analysis assigns a confidence score ranging from $[0,100]$ for each criterion. The last level provides a qualitative assessment of the test script. It attributes for each criterion two membership rates $R_{E B_{-} l}$ and $R_{E B_{-} 2}$ of qualitative evaluation labels among very well (VW), Well (W), medium (M), bad (B), very bad (VB). Therefore, the membership rates $R_{E B_{-} I}$ and $R_{E B_{-} 2}$ are computed according the projection of the average value of the criterion scores $S_{\text {Crit }}$ provided at the input of the decision fusion stage (see Fig.1), on the intersected linguistic fuzzy subsets among the five labels (see Fig.12). 


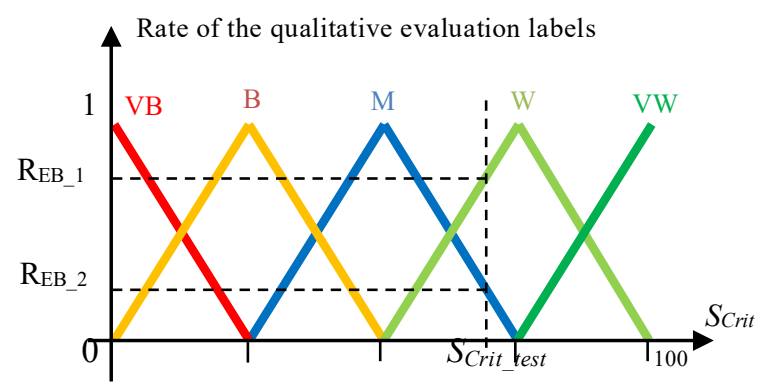

Fig. 12. Qualitative evaluation labels

Table 2. Overall CCR [\%] using correct/ incorrect samples of shape and direction criteria.

\begin{tabular}{lcccc|ccc}
\hline Criteria & \multicolumn{4}{c|}{ Shape } & \multicolumn{3}{c}{ Direction } \\
\cline { 2 - 8 } \multicolumn{1}{c}{ Models } & BEM & FDM & CNN & Fusion & BEM & FDM & Fusion \\
\hline Arabic characters & 96,56 & 97,16 & 98,86 & 99,02 & 98,16 & 96,26 & 98,71 \\
Urdu characters & 95.25 & 94.65 & 97.04 & 97.50 & 99.20 & 97.18 & 99.20 \\
Persian characters & 93.40 & 93.93 & 94.33 & 94.46 & 97.98 & 97.11 & 98.13 \\
Arabic words & 94,13 & 96,50 & 97,25 & 97,96 & 97,10 & 96,13 & 98,61 \\
Latin characters & 96,9 & 97,02 & 97,14 & 97,92 & 99,50 & 97.11 & 99,50 \\
Symbol & 97,13 & 98,02 & 99,11 & 99,41 & 98.13 & 98.08 & 99.12 \\
\hline
\end{tabular}

Table 3. Overall CCR [\%] using correct/ incorrect samples of order, position, and kinematic criteria.

\begin{tabular}{|c|c|c|c|c|c|c|c|}
\hline \multirow{2}{*}{$\begin{array}{l}\text { Criteria } \\
\text { Models }\end{array}$} & \multicolumn{3}{|c|}{ Order } & \multirow{2}{*}{$\begin{array}{c}\text { Kinematic } \\
\text { BEM }\end{array}$} & \multicolumn{3}{|c|}{ Position } \\
\hline & BEM & FDM & Fusion & & BEM & $\mathrm{CNN}$ & Fusion \\
\hline Arabic characters & 98,75 & 97,15 & 98,75 & 98,75 & 99.00 & 97,06 & 99.00 \\
\hline Urdu characters & 99.02 & 98.11 & 99.02 & 98.00 & 97.19 & 96.15 & 97.85 \\
\hline Persian characters & 97.33 & 97.81 & 98.15 & 97.25 & 96.82 & 96.28 & 97.44 \\
\hline Arabic words & 97,62 & 95,35 & 97,84 & 97,20 & 95,77 & 93,16 & 96.11 \\
\hline Latin characters & 97.30 & 96.14 & 97,72 & 98.90 & 96,51 & 95,18 & 97.13 \\
\hline Symbol & 98.20 & 96.10 & 99,15 & 98,60 & 98,62 & 98,11 & 99.97 \\
\hline
\end{tabular}

\subsubsection{Global analysis}

Before presenting the detail of quantitative and qualitative results of handwriting analysis, it is necessary to provide an overall legibility assessment of each criterion. It involves an evaluation of the test scripts classification process applied by each model. In this process, FDM and CNN approaches operate SVM algorithms, while the global classification using BEM approach assigns the test sample to the class of index $i_{c}$ following the eq. 14 .

$$
i_{c}=\operatorname{argmax}\left\{S_{\text {shape }}, 1-S_{\text {direction }}, 1-S_{\text {ordre }}, 1-S_{\text {kinematic }}, 1-S_{\text {position }}\right\}
$$

Where: $S_{\text {crit: }}$ is the score of the correspondent criterion.

Tables 2, and 3 summed up the global results achieved by our system using the different feature extraction models and their fusion. We can see from these tables that our method is very effective for handwriting children's evaluation. In fact, it gives good results for characterizing the five criteria. The specific temporal information and geometric parameters provided by BEM allow us to analyze the five criteria (shape, direction, order, kinematic, and position). FDM is used to characterize (shape, direction, order) regardless of the handwriting velocity. Finally, the generic features generated by CNN allowing to apprehend only the shape and position criteria regardless of the handwriting dynamic and order aspects. 
Table 4. Quantitative results of some Arabic, Latin, Persian, Urdu scripts and symbols using shape, order and direction criteria.

\begin{tabular}{|c|c|c|c|c|c|c|c|}
\hline \multirow{2}{*}{$\begin{array}{l}\text { Criteria } \\
\text { Model }\end{array}$} & \multicolumn{3}{|c|}{ Shape } & \multicolumn{2}{|c|}{ Order } & \multicolumn{2}{|c|}{ Direction } \\
\hline & BEM & FDM & CNN & BEM & FDM & BEM & FDM \\
\hline & \multicolumn{7}{|c|}{ Arabic characters } \\
\hline i & 0.95 & 0.97 & 0.99 & 0.99 & 0.95 & 1.00 & 0.97 \\
\hline ب & 0.94 & 0.97 & 0.98 & 0.98 & 0.96 & 0.99 & 0.95 \\
\hline$\varepsilon$ & 0.95 & 0.95 & 0.97 & - & - & 0.97 & 0.95 \\
\hline$\tau$ & 0.96 & 0.96 & 0.99 & - & - & 0.99 & 0.98 \\
\hline 2 & 0.98 & 0.96 & 0.99 & - & - & 1.00 & 0.97 \\
\hline 9 & 0.97 & 0.97 & 0.98 & - & - & 1.00 & 0.99 \\
\hline$J$ & 0.98 & 0.97 & 0.99 & - & - & 1.00 & 0.97 \\
\hline ص & 0.94 & 0.96 & 0.97 & - & - & 0.97 & 0.93 \\
\hline$b$ & 0.95 & 0.95 & 0.95 & 0.97 & 0.93 & 0.96 & 0.95 \\
\hline س & 0.90 & 0.92 & 0.93 & - & - & 0.93 & 0.90 \\
\hline ن & 0.93 & 0.96 & 0.98 & 0.95 & 0.92 & 0.95 & 0.95 \\
\hline 5 & 0.94 & 0.95 & 0.99 & 1.00 & 1.00 & 1.00 & 0.96 \\
\hline ف & 0.91 & 0.91 & 0.92 & 0.99 & 0.96 & 0.97 & 0.97 \\
\hline ي & 0.88 & 0.94 & 0.96 & 0.96 & 0.94 & 0.96 & 0.97 \\
\hline i & 0.90 & 0.91 & 0.95 & - & - & 1.00 & 1.00 \\
\hline \multirow[t]{2}{*}{$\ddot{b}$} & 0.93 & 0.96 & 0.96 & 0.99 & 0.98 & 1.00 & 0.97 \\
\hline & \multicolumn{6}{|c|}{ Arabic words } & \\
\hline قرأ & 0.89 & 0.92 & 0.95 & 1.00 & 0.93 & 1.00 & 0.95 \\
\hline مدرسة & 0.92 & 0.93 & 0.96 & 0.97 & 0.94 & 0.98 & 0.92 \\
\hline طفل & 0.94 & 0.96 & 0.97 & 0.96 & 0.93 & 0.99 & 0.91 \\
\hline \multicolumn{8}{|c|}{ Urdu and Persian characters } \\
\hline 5 & 0.93 & 0.94 & 0.95 & 0.98 & & 0.96 & 0.98 \\
\hline$j$ & 0.95 & 0.96 & 0.95 & 0.98 & & 0.96 & 0.98 \\
\hline$\varphi$ & 0.92 & 0.94 & 0.96 & 0.98 & & 0.99 & 0.99 \\
\hline$\frac{1}{j}$ & 0.90 & 0.92 & 0.92 & 0.93 & & 0.95 & 0.95 \\
\hline \multicolumn{8}{|c|}{ Latin characters and symbols } \\
\hline A & 0.94 & 0.96 & 0.98 & 0.98 & 0.96 & 0.99 & 0.97 \\
\hline $\mathrm{C}$ & 0.97 & 0.97 & 0.99 & - & - & 1.00 & 0.99 \\
\hline D & 0.93 & 0.96 & 0.98 & 0.99 & 0.97 & 1.00 & 0.95 \\
\hline$E$ & 0.95 & 0.94 & 0.97 & 1.00 & 0.95 & 0.99 & 0.97 \\
\hline $\mathrm{H}$ & 0.96 & 0.96 & 0.98 & 0.99 & 0.97 & 1.00 & 0.97 \\
\hline$\$$ & 0.98 & 0.98 & 1.00 & 0.99 & 0.97 & 1.00 & 0.98 \\
\hline$\&$ & 0.96 & 0.96 & 0.98 & - & - & 1.00 & 1.00 \\
\hline
\end{tabular}

\subsubsection{Quantitative analysis}

The accuracy $A S_{\text {accuracy }}$ of the quantitative evaluation obtained by the different individual approaches implemented in the proposed system is described in Eq.15. It is computed by comparing the provided scores $A S_{\text {predicted }}$ at the output of their respective modules with the scores $A S_{\text {expected }}$ assigned by the teachers during the database collection.

$$
A S_{\text {accuracy }}=1-\left|A S_{\text {predicted }}-A S_{\text {expected }}\right|
$$

We evaluate the performance of our handwriting analysis system for all supported sequences (Arabic, Urdu, Persian, and Latin letters, Arabic words, and symbols) on the collected test dataset. The obtained quantitative results of some observations are shown in Tables 4 and 5. It shows that the best confidence score of shape criterion is achieved by using CNN and FDM rather than BEM (see Table 4). This can be explained by the use of visual and curvature generic features generated by CNN and FDM respectively. Also, the $\mathrm{BEM}$ approach is more accurate for the analysis of order and direction criteria than a network of score regression or prediction like the association FDMSVM. This robustness is due to the precise features to characterize the change of direction and order of elementary strokes. We notice also that the kinematic criterion allows us to distinguish the nature of writing (fast or slow) based on a powerful BEM model (see Table 5). This is due to the use of dynamic parameters that give the overall temporal properties of the neuromuscular networks implicated in motion generation, and the geometric properties of all the muscles and joints inducted to execute the movement. Likewise, we can see from this table that the obtained results using BEM and $\mathrm{CNN}$ for position of the trace criterion are very powerful which aid children during the learning process. 
Table 5. Quantitative results of some test samples of Arabic, Latin, Persian, Urdu scripts and symbols using position, and kinematic criteria.

\begin{tabular}{|c|c|c|c|}
\hline Criteria & \multicolumn{2}{|c|}{ Position of the trace } & \multirow{2}{*}{$\frac{\text { Kinematic }}{\text { BEM }}$} \\
\hline Model & BEM & CNN & \\
\hline i & 0.95 & 0.97 & 0.99 \\
\hline ب & 0.94 & 0.91 & 0.98 \\
\hline$\varepsilon$ & 0.95 & 0.95 & 0.99 \\
\hline$\tau$ & 0.96 & 0.91 & 0.92 \\
\hline د & 0.98 & 0.96 & 0.97 \\
\hline 9 & 0.97 & 0.97 & 0.98 \\
\hline$\jmath$ & 0.98 & 0.97 & 0.98 \\
\hline ص & 0.94 & 0.96 & 0.91 \\
\hline$b$ & 0.95 & 0.95 & 0.88 \\
\hline س & 0.93 & 0.91 & 0.87 \\
\hline ن & 0.93 & 0.96 & 0.95 \\
\hline ك5 & 0.94 & 0.95 & 0.83 \\
\hline ف & 0.91 & 0.91 & 0.96 \\
\hline ي & 0.93 & 0.95 & 0.96 \\
\hline 5 & 0.90 & 0.91 & 0.89 \\
\hline$\ddot{0}$ & 0.93 & 0.96 & 0.99 \\
\hline 3 & 0.94 & 0.94 & 0.97 \\
\hline$j$ & 0.96 & 0.94 & 0.97 \\
\hline 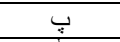 & 0.93 & 0.92 & 0.97 \\
\hline$\frac{1}{5}$ & 0.90 & 0.89 & 0.95 \\
\hline قرأ & 0.96 & 0.98 & 1.00 \\
\hline مدرسة & 0.92 & 0.93 & 0.97 \\
\hline طفل & 0.94 & 0.96 & 0.96 \\
\hline $\mathrm{A}$ & 0.94 & 0.96 & 0.98 \\
\hline $\mathrm{C}$ & 0.97 & 0.92 & 0.97 \\
\hline $\mathrm{D}$ & 0.96 & 0.98 & 0.99 \\
\hline$E$ & 0.95 & 0.94 & 1.00 \\
\hline $\mathrm{H}$ & 0.96 & 0.96 & 0.99 \\
\hline$\$$ & 0.98 & 0.98 & 0.99 \\
\hline$\&$ & 0.96 & 0.96 & 1.00 \\
\hline
\end{tabular}

\subsubsection{Qualitative analysis}

As illustrated in Fig. 13, Fig. 14, and Fig. 15, qualitative assessments of the used criteria are presented respectively for some samples of Arabic, Latin, Urdu, Persian scripts, and symbols. As shown in Fig.11 (a), the test samples label, their number, and their global confidence scores are displayed on the top left corner, right and left bottom corners respectively. Five levels of qualitative assessment are considered for the confidence score: VW (green), W (dark green), M (blue), B (orange), and VB (red) corresponding to a uniform partition of confidence ranges.

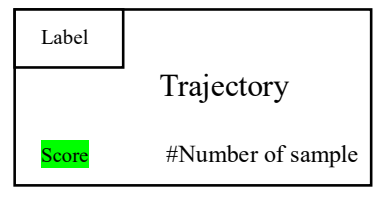

(a) Legend

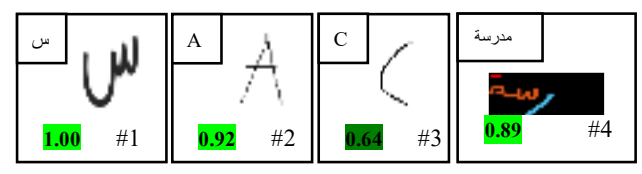

(b) Correct shape

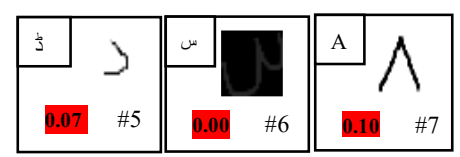

(c) Strokes omission

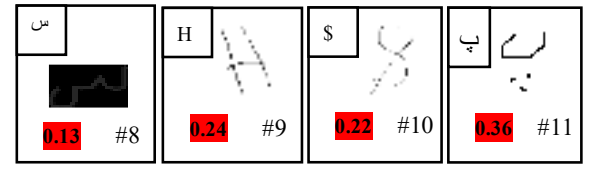

(d) Deformation

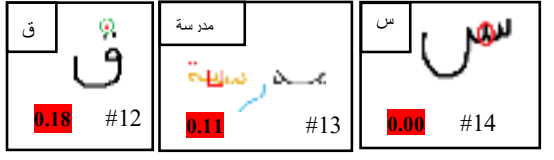

(e) Exceeding stroke

Fig. 13. Qualitative evaluation labels on the shape criterion with a legend (a), correct shape (b), wrong shape due to omission strokes (c), deformation stroke (d), and exceeding stroke (e). 
Results show that the proposed method is rather generic, which gives pertinent analysis scores for both cursive and non-cursive children's handwriting. As depicted in Fig 13.b, it is able to determine the correct shape of some samples $\# 1$ to \#4 having scores greater or equal to 0.5 . It allows the detection of wrong shapes caused by strokes omission (Urdu letter ' 's' \#5, Arabic letter ' $\omega$ ' \#6, and Latin letter ' $A$ ' \#7 of Fig.13.c), strokes deformation (the samples of number \#8, $\# 9,10$ and \#11 of Fig.13.d), or exceeding strokes (The red bounding box in observations \#12, \#13, and \#14 of Fig.13.d) shows instances of the exceeding stroke of the Arabic letter 'ق', the Arabic word 'مدرسة', and Arabic letter 'w' respectively).

Also, qualitative results demonstrate the ability of the system to analyze correctly the direction and order criteria. Fig.14.a) and Fig.14.b) show the evaluation of various numbers of samples according to these criteria. Samples $\# 15$ and \#16 are written in reverse direction while sample \#17 is written in the correct direction. Likewise, samples $\# 18$ and $\# 19$ are written in reverse order, while sample \#20 in the correct order. We notice that the mentioned rate represents the average score attributed to the analyzed sample according to the considered criteria (direction / order) which result from the fusion of the used models. And the color of its highlight represents the qualitative interpretation.

Furthermore, the combination method allows us to analyze the handwriting trajectory kinematics and the respect of the handwriting reference lines. Fig 15.a) shows the difference between the BEM dynamic profiles modeling and their results of samples' analysis that are written quickly (\#21 of Arabic letter 'ص', \#23 of Arabic letter ' $و$ ') to others carefully (\#22). Similarly, the cases of the trace position errors corresponding to the overflow of the reference line have been penalized comparing to those traced with respect to the baseline in terms of criterion score and its qualitative interpretation (color of score highlight, see Fig. 15 b). We can see from this figure that sample \#24 and \#26 has a good score because it is written upon the baseline, as opposed to sample\#25 which is written under the baseline.

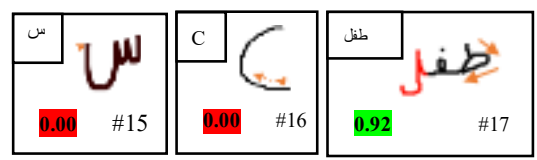

(a) Direction

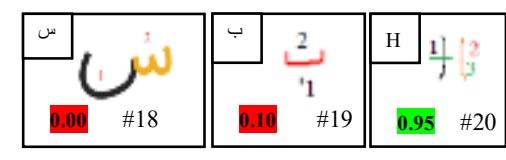

(b) Order

Fig. 14. Qualitative results on both direction and order criteria.

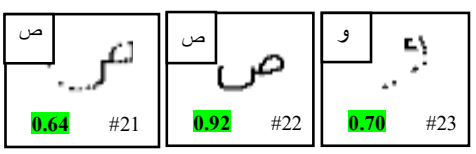

(a) Kinematic

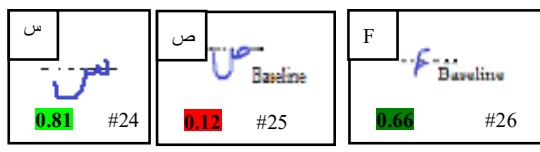

(b) Position of the trace

Fig. 15. Qualitative results on Kinematic and position criteria.

\subsection{Waiting time}

We measure the waiting time (ms) response of the different analysis by changing the number of strokes. As shown in Fig. 16, the waiting time analysis using SVM engine is slightly weak than BEM \& SD-DD method. Also, it seems that the waiting time of the proposed framework is around $1170 \mathrm{~ms}$ for the worst case using 20 strokes. It proves that our model is quite fast and has a lower waiting time for online handwriting analysis. 


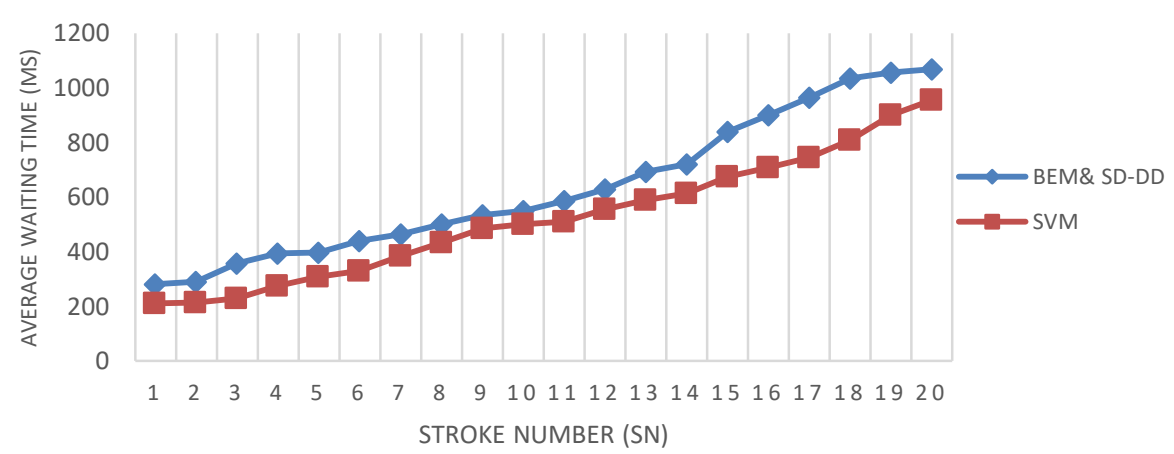

Fig. 16. Waiting time response of analysis models.

\subsection{Discussion}

We discussed below the strengths and limitations of the present framework as well as the comparative performance analysis with other existing online handwriting analysis systems.

\subsubsection{Strengths of the present framework}

Our experimental results show that the proposed analysis criteria and adopting extractor models provide superior performance over existing online Latin handwriting analysis systems. The major strengths of our framework are as following:

- The ability to analyze both cursive and non-cursive multilingual scripts based on the complementarity of feature extraction models.

- The present framework can evaluate the children's handwriting quality with different writing styles such as presented in Fig.17.a) and Fig. 17.b) for correct shape of Arabic Letters ' $\varepsilon$ ' and ' $\tau$ ', Fig.17.c) and Fig. 17.d) of Latin letters ' $a$ ' and ' $i$ ' respectively.

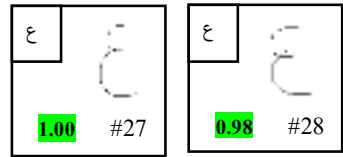

(a) Arabic Letter Ain ' $\varepsilon$ '

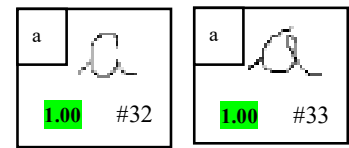

(c) Latin letter ' $a$ '

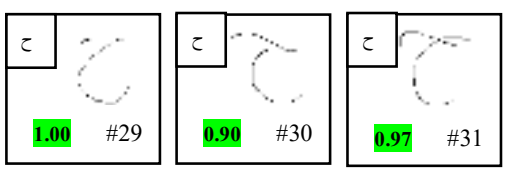

(b) Arabic Letter Haa ' $ح$ '

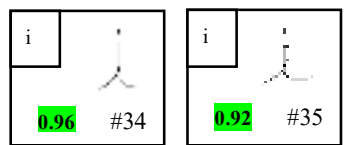

(d) Latin letter 'i'

Fig. 17. Handwriting evaluation quality with different writing styles.

- Our proposal is original compared to other existent researches, since it is based on the combination of two global evaluation approaches: the first one employs SD-DD comparison engine based on BEM with three model samples and the six mentioned classes. The second approach employs the hybrid of FDM and deep learning CNN features with SVM engine.

\subsubsection{Limitation}

While analyzing the limitations of the proposed framework in the handwriting quality analysis system, it was noted that most of the errors generated are mainly due to a misjudgment of the shape criterion corresponding to the BEM approach when the user writes very quickly or slowly. In fact, in these two cases, the BEM generates an irregular number of strokes very different from the model samples, 
which affects the geometry of the strokes and thus the score of the shape criterion. On the other hand, the two modules FDM and CNN compensate for this drawback at the level of the fusion step. Indeed, in an unconstrained context, the expert can readjust the weights of the different criteria in the final decision by increasing the weight of the offline layout script and reducing that of the dynamics. However, the BEM approach is a more appropriate module for the dynamic criterion evaluation.

\subsubsection{Comparative performance analysis}

In this section, we compare the performance of the proposed analysis model using different deep learning architectures as well as with systems in the literature. Table 6 shows the CCR obtained results using different deep learning architecture. We can see from this Table, that the results obtained by CNN with 15 layers and Alex-Net are very competitive for analyzing shape and position criteria. To evaluate direction, order, and kinematic criteria, we have compared the results obtained by BEM \& SD-DD and BEM-LSTM analyzers. The results show the superiority of BEM features and SD-DD algorithm for characterizing direction, order, Kinematic criteria than BEM-LSTM model.

Table 6. Overall CCR [\%] comparison using different deep learning architecture for criteria analysis.

\begin{tabular}{cccccc}
\hline Criteria & \multicolumn{5}{c}{ Analyzer } \\
\cline { 2 - 6 } & Alex-Net & Inception V4 & CNN & $\begin{array}{c}\text { BEM \& } \\
\text { SD-DD }\end{array}$ & $\begin{array}{c}\text { BEM- } \\
\text { LSTM }\end{array}$ \\
\hline Shape & 98,51 & 97,66 & 98,86 & 96.56 & 96.98 \\
\hline Direction & - & - & - & 98.16 & 98.02 \\
\hline Order & - & - & - & 98.75 & 97.25 \\
\hline Kinematic & - & - & - & 98.75 & 95.00 \\
\hline Position & 96,96 & 95,12 & 97,14 & 99.00 & 96.45 \\
\hline
\end{tabular}

As already mentioned, there are no available researches for the analysis of online Arabic handwriting script as there are very few significant studies on Latin script in the literature. All the existing analytical works in Latin script have used different datasets that are not always available, so these studies are not comparable directly. This did not prevent us to compare the performance of the proposed framework with these existing studies [1], [3], [16] to get an idea of comparative performance analysis.

We retain that the performance of our system in Latin script is comparable to that of the reference commercial systems [3], and [16], in the order criterion with $97.62 \%$ of CCR and a slightly advantageous in terms of correct analysis for shape and direction criteria with $96.6 \%$ and $98.3 \%$ of CRR respectively. On the other hand, our system is distinguished by the possibility of analyzing other essential criteria such as kinematic (dynamics) and respect of reference lines which are two important aspects in the learning step.

\subsection{Overview of Recognition system}

In order to highlight a practical application, we have also integrated a recognition model. It allows to automatically assign the test script to the appropriate label existing in the system database. The architecture of our recognition system is based on the combination of BEM and CNN for handwritten feature extraction, and DBLSTM with SVM classifiers [40]. It has four modules: a preprocessing module for denoising the online handwriting trajectory. BEM and CNN models are employed to extracts two types of features vector from the online and offline trajectories respectively. The online features are studied using DBLSTM whereas the offline on SVM classifier. The last step combines the two modules in order to increase the discrimination power of the overall system. 


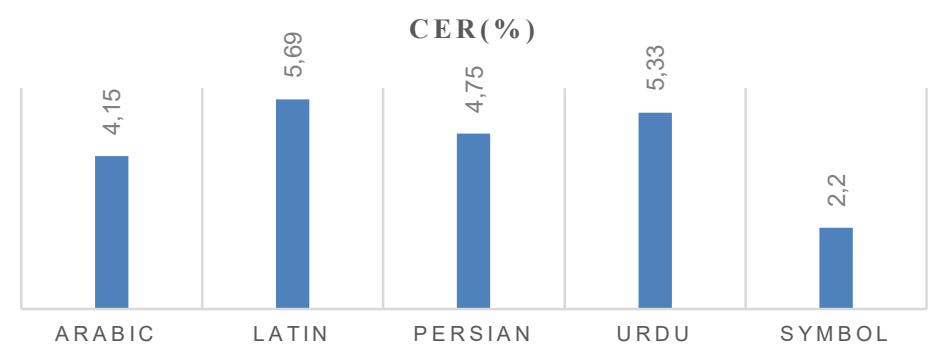

Fig. 18. CER of proposed architecture using mu.

To validate the performance of the proposed model on online handwriting recognition using a multi-script constructed database, we have used Character Error Rate (CER) metric. As presented in Fig. 18, the obtained CER results show also the effectiveness of our proposed model in online handwriting recognition topics. Thus, we observe that our proposed features extraction models on the one hand, and the use of deep learning classifier, on the other hand, are useful and effective for online handwriting recognition in a multi-lingual context.

\section{Conclusion and Future Work}

This paper introduces a new analysis method for children handwriting quality allowing them to deal with both cursive and non-cursive handwriting multiscript. The key emphasis of our project is to analyze the handwriting quality of school children and provide them with relevant real-time feedback based on multiple criteria. The proposed system uses the combination of three models for handwriting representation. The BEM is characterized by the strong ability to analyze the five main criteria (shape, direction, order, kinematic, position). The FDM offers a specific feature allowing the precise analysis of shape, order, and direction criteria. Also, $\mathrm{CNN}$ is employed to describe the final shape of the post-drawing view.

The evaluation results and the computed scores assigned for each criterion are conducted using two different methods: SD-DD and SVM-based comparison engines. The efficiency of the single corresponding models, and the combination thereof, were evaluated in experiments using different observations of the broad database.

We have also observed that the used extracted features are rather generic and their applicability in other scripts as Chinese, Telugu, English, etc., is interesting. Also, we maintain that our proposal can be appropriated for other applications such as the pre-diagnosis of neuromuscular pathologies, the difficulties of dyslexia and Parkinson's disease, so we envisage it as future works.

\section{Acknowledgements}

The research leading to these results has received funding from the Ministry of Higher Education and Scientific Research of Tunisia under the grant agreement number LR11ES4.

\section{References}

1. Jolly C, Palluel-Germain, R, Gentaz, E., (2013) Evaluation of a tactile training for handwriting acquisition in french kindergarten children: A pilot study, Kindergartens: Teaching methods, expectations and current challenges 161-176.

2. Bonneton-Botté, N. (2020): Can tablet apps support the learning of handwriting? An investigation of learning outcomes in kindergarten classroom. Comput. Educ. 38

3. Simonnet, D., Girard, N., Anquetil, D., Renault, M. E., Thomas, S. (2018). Evaluation of Children Cursive Handwritten Words for e-Education. Pattern Recognition Letters, doi: 10.1016/j.patrec.07.021/

4. Fan, Y, Sakriani S, Yang W, and Satoshi N. (2019) Make skeleton-based action recognition model smaller, faster and better. Proceedings of the ACM Multimedia Asia.

5. Jun, L, Amir S., Gang W, Ling-Yu D., and Alex Kot. (2019) Skeleton-based 
online action prediction using scale selection network. IEEE Transactions on Pattern Analysis and Machine Intelligence, PP, 02.

6. Z. Xie, Z. Sun, L. Jin, H. Ni, T. Lyons, (2017) "Learning spatial-semantic context with fully convolutional recurrent network for online handwritten text recognition," to appear in IEEE Trans. on PAMI.

7. Sen, S., Shaoo D., Paul S., Sarkar R., Roy K..: (2018) Online Handwritten Bangla Character Recognition Using CNN: A Deep Learning Approach. In: Advances in Intelligent Systems and Computing, pp 413-420, April.

8. Y. C. Wu, F. Yin, C. L. Liu, (2017) "Improving handwritten Chinese text recognition using neural network language models and convolutional neural network shape models," Pattern Recognition, Vol. 65, pp.251-264.

9. Lecun, Y. and Bengio, Y. (1998) "Convolutional Networks for Images, Speech, and Time-Series," Handb. Brain Theory Neural Netw., pp. 255-258.

10. Dinehart, L. H. (2015) Handwriting in early childhood education: Current research and future implications, Journal of Early Childhood Literacy 97118.

11. Rosenblum, S., Weiss, P.L., Parush, S., (2003). Product and process evaluation of handwriting difficulties. Educational Psychology Review 15, 41-81.

12. Guinet, E. S. Kandel, Ductus (2010). A software package for the study of handwriting production, Behavior Research Methods 326-332.

13. Accardo, A. P., Genna, M., Borean, M. (2013). Development, maturation and learning influence on handwriting kinematics, Human movement science $136-146$.

14. Hu, Z.-H. Xu, Y., L.-S. Huang, H. Leung, (2009). A Chinese handwriting education system with automatic error detection, Journal of Software 101 107.

15. Falk, T. H. Tam C., Schellnus, H. Chau, T. (2011). On the development of a computer-based handwriting assessment tool to objectively quantify handwriting proficiency in children, Computer Methods and Programs in Biomedicine 102-111.

16. Simonnet, D., Girard, N., Anquetil, D., Renault, M. E., Thomas, S. (2018). Evaluation of Children Cursive Handwritten Words for e-Education. Pattern Recognition Letters, doi: 10.1016/j.patrec.07.021.

17. Simon, C., Elisa, F., Eric, A, Pauline N. (2020) Integrating Writing Dynamics in CNN for Online Children Handwriting Recognition. 17th International Conference on Frontiers in Handwriting Recognition (ICFHR).

18. Krichen O., Corbillé S., Anquetil E., Girard N., Nerdeux P. (2021) Online Analysis of Children Handwritten Words in Dictation Context. In: Barney Smith E.H., Pal U. (eds) Document Analysis and Recognition, ICDAR Workshops. ICDAR, Lecture Notes in Computer Science, vol 12916. Springer, Cham. https://doi.org/10.1007/978-3-030-86198-8_10

19. McAuley, J. H., C. D. Marsden Brain. (2000) Physiological and pathological tremors and rhythmic central motor control ", Volume 123, Issue 8, Pages $1545-1567$

20. Boubaker, H., Chaabouni, A. Tagougui, N., Kherallah M. and Adel M. Alimi, (2013) "Handwriting and Hand drawing Velocity Modeling by Superposing Beta Impulses and Continuous Training Component," International Journal of Computer Science Issues (IJCSI), Vol. 10, Issue 5, pp $57-63$.

21. Bezine, H., Adel M. Alimi, S. Nasser, (2004) "Generation and analy-sis of handwriting script with the betaelliptic model", In the proceeding of the 9th International Workshop on Frontiers in Handwriting Recognition, IWFHR9,2004 , pp. $515-520$.

22. Plamondon, R. (1995). A kinematics theory of rapid human movements. Part I: Movement representation and generation. Biological Cybernet. 72, 295 307.

23. Plamondon R., O'Reilly C., Galbally J., Almaksour A., Anquetil E., (2014). Recent developments in the study of rapid human movements with the kinematic theory: Applications to handwriting and signature synthesis", Pattern Recognition Letter, Vol. 35, pp. 225 - 235.

24. Alimi, M., (1997). An evolutionary neuro-fuzzy approach to recognize online Arabic handwriting, in: Document Analysis and Recognition, Proceedings of the Fourth International Conference on, IEEE. pp. 382-386. 
25. Hamdi., Y., Boubaker H., Dhieb., T., Elbaati., A, Alimi., A. (2019). Hybrid DBLSTM-SVM based Beta-elliptic-CNN Models for Online Arabic Characters Recognition. In International Conference on Document Analysis and Recognition (ICDAR), pages 803-808.

26. Hamdi Y., Boubaker H., Alimi A.M. (2021) Online Arabic Handwriting Recognition Using Graphemes Segmentation and Deep Learning Recurrent Neural Networks. In: Hassanien A.E., Darwish A., Abd El-Kader S.M., Alboaneen D.A. (eds) Enabling Machine Learning Applications in Data Science. Algorithms for Intelligent Systems. Springer, Singapore. https://doi.org/10.1007/978-981-33-6129-4_20

27. Dhieb, T., Rezzoug, N., Boubaker, H., Gorce, P., and A. M. Alimi, (2019) "Effect of age on hand drawing movement kinematics," Computer Methods in Biomechanics and Biomedical Engineering, Vol. 22 Issue sup1: 44th, DOI:10.1080/10255842.2020.1714235.

28. Rabhi, B., Elbaati, A., Boubaker, H. et al. (2021) Multi-lingual character handwriting framework based on an integrated deep learning based sequence-to-sequence attention model. Memetic Comp. https://doi.org/10.1007/s12293-021-00345-6

29. Rabhi B., Elbaati A., Hamdani T.M., Alimi A.M. (2021) ASAR Competition on Online Signal Restoration Using Arabic Handwriting Dhad Dataset. In: Barney Smith E.H., Pal U. (eds) Document Analysis and Recognition ICDAR 2021 Workshops. ICDAR 2021. Lecture Notes in Computer Science, vol 12916. Springer, Cham. https://doi.org/10.1007/978-3-03086198-8 26

30. Dhieb, T., Ouarda, W., Boubaker, H., and A. M. Alimi, (2016). "BetaElliptic Model for Writer Identification from Online Arabic Handwriting", J. of Information Assurance and Security, ISSN: 1554-1010, Volume 11, Issue 5, pp. 263-272.

31. Persoon E. and Fu K. S., (1986). "Shape Discrimination using Fourier Descriptors," Journal IEEE Transactions on Pattern Analysis and Machine Intelligence, pp. $388-397$.

32. Krizhevsky, A., and Sutskever, Ilya and Hinton, Geoffrey E. (2012) "Imagenet classification with deep convolutional neural networks. Advances in neural information processing systems:10971105.

33. Simonyan, K., Zisserman, A., (2014) "Very deep convolutional networks for large-scale image recognition". ArXiv.

34. Szegedy, C., Ioffe, S., Vanhoucke, V., and Alemi, A. (2017) "Inception-v4, Inception-ResNet and the Impact of Residual Connections on Learning." AAAI.

35. Zhang, X.-Y. Bengio, Y. C.-L. Liu, (2017). Online and offline handwritten chinese character recognition: A comprehensive study and new benchmark, Pattern Recognition 61 348-360.

36. Zouari, R., Boubaker, H., Kherallah, M.,: (2018) "Multi-language online handwriting recognition based on beta-elliptic model and hybrid TDNN-SVM classifier." Multimedia Tools and Applications 78: 12103-12123.

37. Bernhard, E., Boser, Isabelle M Guyon, and Vladimir N Vapnik. (1992) A training algorithm for optimal margin classifiers. In Proceedings of the fifth annual workshop on Computational learning theory, pages 144-152. ACM.

38. Hamdi, Y., Boubaker, H. \& Alimi, A.M. (2021) Data Augmentation using Geometric, Frequency, and Beta Modeling approaches for Improving Multilingual Online Handwriting Recognition. IJDAR 24, 283-298. https://doi.org/10.1007/s10032-021-00376-2

39. Platt, J., (1999) "Probabilistic outputs for support vector machines and comparison to regularized likelihood methods". In Advances in Large Margin Classifiers. MIT Press.

40. Hamdi Y., Boubaker H., Hamdani T.M., Alimi A.M. (2021) ASAR 2021 Competition on Online Arabic Character Recognition: ACRC. In: Barney Smith E.H., Pal U. (eds) Document Analysis and Recognition - ICDAR 2021 Workshops. ICDAR 2021. Lecture Notes in Computer Science, vol 12916. Springer, Cham. https://doi.org/10.1007/978-3-030-86198-8_27 